\title{
Improved time to treatment failure and survival in ibrutinib-treated malignancies with a pharmaceutical care program: an observational cohort study
}

\author{
Jeremie Zerbit ${ }^{1}$. Sylvie Chevret ${ }^{2,3} \cdot$ Sophie Bernard ${ }^{4} \cdot$ Marie Kroemer $^{5} \cdot$ Charlotte Ablard $^{1} \cdot$ Stephanie Harel $^{6}$. \\ Pauline Brice $^{4}$ • Isabelle Madelaine ${ }^{1}$ • Catherine Thieblemont ${ }^{3,4}$
}

Received: 31 January 2020 / Accepted: 15 April 2020 / Published online: 1 June 2020

(C) The Author(s) 2020

\begin{abstract}
Ibrutinib treatment has been shown to increase survival in patients with B cell malignancies. Real-life data suggest a large part of discontinuations are due to toxicities, impairing ibrutinib efficacy. We aimed to assess the impact of a pharmaceutical care program on the efficacy and safety of ibrutinib. This single-center, cohort, observational study enrolled patients with B cell malignancies. Patients were either assigned to the program or to receive usual care, based on physician decision. The program was conducted by clinical pharmacists specializing in oncology and included patient education for management of toxicities, adherence monitoring, interventions to reduce drug-drug interactions, and follow-up of transition from hospital to community. Between February 2014 and May 2017, we enrolled 155 patients, including 42 (27\%) who were allocated to the program group and $113(73 \%)$ to the usual care group. The effect of the program was beneficial in terms of time to treatment failure $(p=0.0005)$. The 30-month progression-free and overall survivals were significantly superior in the program group (respectively $p=0.002$ and $p=0.004)$. Grade 3 or higher adverse events occurred more frequently for patients in the usual care group (15\%) than program group (8\%). A pharmaceutical care program provides a personalized environment for outpatient monitoring and control of the key risks associated with oral anticancer agents. This study shows evidence that management of ibrutinib treatment by clinical pharmacists results in significant improvement in survival and better tolerance than usual care.
\end{abstract}

Keywords Ibrutinib $\cdot$ Leukemia $\cdot$ Lymphoma $\cdot$ Survival $\cdot$ Pharmacist $\cdot$ Education

Electronic supplementary material The online version of this article (https://doi.org/10.1007/s00277-020-04045-y) contains supplementary material, which is available to authorized users.

Catherine Thieblemont catherine.thieblemont@aphp.fr

1 Pharmacy Department, APHP, Saint-Louis Hospital, Paris, France

2 Department of Biostatistics and Medical Information, APHP, Saint-Louis Hospital, Paris, France

3 Paris Diderot University, Sorbonne Paris-Cité, Paris, France

4 Hemato-Oncology Department, APHP, Saint-Louis Hospital, 1, Avenue Claude Vellefaux, 75010 Paris, France

5 Pharmacy Department, University Hospital Center of Besançon, Besançon, France

6 Immuno-Hematology Department, APHP, Saint-Louis Hospital, Paris, France

\section{Introduction}

In the last two decades, major advances in understanding the biology of hematological malignancies have led to significant progress in their treatment. In particular, an increasing amount of oral anticancer agents (OAA) have demonstrated remarkable outcomes in patients.

Ibrutinib is a first-in-class, once-daily, oral inhibitor of Bruton tyrosine kinase (BTK) approved for the therapy of B cell malignancies including chronic lymphocytic leukemia (CLL), Waldenström macroglobulinemia (WM), marginal zone (MZL), and mantle cell lymphoma (MCL). Ibrutinib has demonstrated marked efficacy leading to improved progression-free survival (PFS) and overall survival (OS) among patients, including those with relapsed or refractory disease, and high-risk, elderly, or comorbid patients [1-4].

Benefits of ibrutinib are impaired by its self-administration in the home setting involving challenges of any OAA with 
respect to monitoring of toxicity, adherence, and drug-drug interactions (DDIs).

Despite acceptable tolerance reported in clinical trials, studies evaluating the causes of ibrutinib discontinuations showed that adverse events (AEs) were responsible for $80 \%$ of discontinuations in CLL [5] and of 29\% in MW [6]. Data from real-world use of ibrutinib indicated a $51 \%$ discontinuation rate due to $\mathrm{AEs}$ [7], the most common being atrial fibrillation, infection, pneumonitis, bleeding, and arthralgia. Reviews on OAA indicate that adherence is frequently low, below $80 \%$ of patients [8]. Management of ibrutinib dose adherence appears to be decisive. On the one hand, disadherence is associated to decreased PFS in CLL patients [9], on the other hand, dose reduction because of adverse events allows continuation without affecting PFS [10]. Ibrutinib is metabolized by CYP3A4 and co-administration with strong CYP3A4 inhibitor or inducer is not recommended, while a moderate inhibitor remains possible if absolutely necessary by reducing the dose of ibrutinib to $140 \mathrm{mg}$ daily [11]. Among patients treated with ibrutinib for chronic lymphocytic leukemia, $64 \%$ have co-medications that could increase toxicity by drug interactions including 18\% through CYP3A4 [12].

Managing these risks is essential to keep patients on ibrutinib long enough to achieve an overall response. Pharmaceutical care program (PCP) is a potentially attractive service to improve treatment safety and quality of oral chemotherapy [13]. This study was designed to investigate the effectiveness and safety of a PCP-based management of patients receiving ibrutinib. We hypothesized that the program could impact the PFS by both improving the adherence and reducing the occurrence of adverse events in these patients.

\section{Methods}

\section{Study design and participants}

We conducted a single-center prospective, cohort study to assess effects of a PCP on efficacy and safety of ibrutinib.

Eligible patients were at least 18 years old, diagnosed with chronic lymphocytic leukemia, Waldenström macroglobulinemia, mantle cell lymphoma, or other B cell malignancies with evidence of disease progression requiring ibrutinib treatment according to consensus guidelines. Exclusion criteria were the initiation of ibrutinib treatment within 6 months and the monitoring in another hospital than the study center.

Patients were enrolled at the time of ibrutinib initiation and were either assigned to start the pharmaceutical care program (PCP group) or to receive only usual care without additional monitoring (control, usual care group).

The study protocol was approved by the institutional review board (IRB-00003835) and all patients provided written informed consent for participation in the study. Data collection has been declared to the National Commission for Data
Processing and Freedoms. The study was registered with the French National Agency for Medicines and Health Products Safety (number 2017-A03604-49).

\section{Procedures}

Decision of allocation to the PCP relied on their oncologist. The PCP started in the first 10 days of ibrutinib primo-prescription. Clinical pharmacists specializing in oncology conducted pharmaceutical consultations in day hospital of hematology ward. Patients were seen in PCP every 3 months until the sixth month of treatment, then every 6 months. The PCP involved at least four pharmaceutical consultations lasting between 30 and $60 \mathrm{~min}$. The pharmaceutical consultations were spaced by usual oncological consultations. Patients in the control group received usual care in routine practice including monthly oncologist consultations during 3 months then every 3 months.

The PCP was multimodal and included patient education for self-management in case of toxicities, proactive adherence monitoring, medication-related interventions to reduce drug-drug interactions, and follow-up of transition from hospital to community.

The education step started with a procedure-based evaluation of patient health literacy and fixing of goal setting. Patients were leaving the PCP when goals were met. The training for AEs selfmanagement used take-home information and guidelines for selfmanagement interventions in case of fever and infections, diarrhea, bleeding events, fatigue, cytopenia, and atrial fibrillations. Interventions were suggested according to the severity of toxicities, using a 4-degree scale: no change, taking medication prescribed to take as needed, consulting a general practitioner, and going to emergencies. Whatever the intervention, unless any change, the patient should call either a pharmacist or oncologist. The clinical pharmacist did a telephone follow-up for patients who required an outpatient intervention.

The education aimed to increase patients understanding of the treatment, as well as of its risks, benefits, and proper use. The program required an active role of patients to give them a greater sense of responsibility and warrant an adequate adherence. Adherence was evaluated monthly by using both patient diaries (self-evaluation) and adjudicated prescription claims from pharmacy database. All patient treated by ibrutinib were asked to rate each administration on an institutional monthly diary. The claims data method calculated the medication possession ratio (MPR) defined by the percentage of supply days received divided by the dispensing period. In case of disadherence, the cause was identified and resolved by a reinforcement of the education, a relief of the side effects, or a dose reduction, as appropriate. A familiar caregiver was identified and educated, particularly in case of physical, psychic, or socio-economical barrier.

Medication-related interventions consisted of a medication review for interaction check by clinical pharmacists. Drug interactions were identified and classified as previously described [14] and by analysis of minimum three sources for 
collecting drug prescriptions. In case of DDI qualified as potentially clinically relevant, interventions were proposed by the pharmacist to physicians among a drug addition, withdrawal, substitution, change of administration modality, dosage adjustment, and/or a specialized medical consultation or a biological test.

The follow-up of transition from hospital to community was performed by clinical pharmacists with a formalized transmission of information to general practitioners, community pharmacists and, when appropriate, specialist organ physicians. Community care professionals in charge of patients were informed about starting of ibrutinib and inclusion in the PCP with a standard letter describing the treatment and its monitoring in the PCP. Then, they received a report following each pharmaceutical consultation. The clinical pharmacist communicated any decision leading to change patient care, especially in case of medication-related interventions or management of an adverse effect and/or a poor adhesion.

\section{Outcomes}

The primary efficacy outcome of the study was progressionfree survival (PFS, time from treatment onset to date of progression or death from any cause, whichever occurred first). Secondary outcomes were adherence rates, DDIs, time to treatment failure (TTF), and overall survival (OS, time from treatment onset to date of death). TTF was defined as the time from treatment onset to discontinuation for any reason excluding remission, i.e., disease progression, treatment toxicity, patient preference, or death.

Safety outcomes were adverse events as measured by hematologists during usual consultations and measurement of laboratory variables. Using the NCI Common Terminology Criteria for Adverse Events version 3.0, adverse events were reported monthly and graded on a 0 to 4 scale ( 0 , normal; 4 , life-threatening).

Data were collected monthly from treatment beginning to 6 months, then every 3 months.

\section{Statistical analysis}

A propensity score (PS) approach was used to control for observed confounding factors $[15,16]$. Propensity score was defined as the patient's probability of being allocated to the PCP group, based on the individual observed covariates. Probability was estimated using a logistic regression model with PCP exposure as the dependent variable in relation to the following baseline characteristics: disease, time to treatment, and any other factors selected as associated with the outcome in the cohort. The primary analysis was based on propensity score matching, with a 1:1 matching algorithm without replacement to match exposed and non-exposed subjects on propensity score within a caliper of 0.1 standard deviation of the logit of the propensity score. Imbalance after matching thus was carefully checked using mean standard mean differences and c-index [17]. Once all imbalances have been erased, the outcomes were compared between exposed and non-exposed patients using Cox regression fit with robust variance to account for correlations within the matched pairs [18].

Sensitivity analyses were performed to handle missing covariates. Multiple imputations of missing data was performed, using chained equations, incorporating all baseline variables of the propensity score model, as recommended [19]. Thirty independent imputed datasets were generated with propensity score estimated on each, then averaged before matching.

All tests were 2 -sided, with $P$ values less than 0.05 considered significant. Analyses will be performed on R 3.5.1.

\section{Results}

\section{Patients}

Between February 25, 2014, and May 9, 2017, we assessed 211 patients for eligibility, of whom 155 (73\%) were subsequently enrolled. The most common reason for exclusion was initiation of ibrutinib treatment for less than 6 months. Patients were allocated to the PCP group $(n=42)$ or the usual care group $(n=113)$. Eight participants from the control group missed the 6-month follow-up (Fig. 1).

Table 1 summarizes the differences in treatment groups in the original cohort. Baseline characteristics of patients assigned either in the PCP or in the control group were not significantly different. Across the entire cohort, the median age was 70.3 years (interquartile range (IQR), 63.477.7 years) with $80(51.6 \%)$ patients being 70 years of age or older (Table 1). Patients had undergone a median of two (IQR, 1-3) previous lines of therapy including $63(40.6 \%)$ who received three or more previous lines. The median diagnostic duration before inclusion was 71.3 (IQR, 28.1$122.8)$ months. Main diagnoses were CLL $(n=80,51.6 \%)$, mantle cell lymphoma $(n=32,20.7 \%)$, and Waldenström macroglobulinemia $(n=23,14.8 \%)$. Thirty-two patients had advanced-stage LLC (Rai stage III or IV), among whom $28 \%$ had the high-risk genetic features del(17p) $(n=29)$, del(11q) $(n=9)$, or TP53 mutation $(n=10)$. Five $(16 \%)$ and $18(56 \%)$ patients had a high international prognostic scoring system (IPSS) for WM and a high MCL international prognostic index (MIPI), respectively.

All the 42 patients in the PCP have benefitted of 109 pharmaceutical consultations; all participants accomplished the first step of the PCP, and 13 (31\%) achieved the four consultations of the PCP. The median follow-up was 13.8 (IQR, 7.619.8) months and 92 (59\%) patients were still receiving ibrutinib at the time of last follow-up. 
Fig. 1 Patient flow and disposition

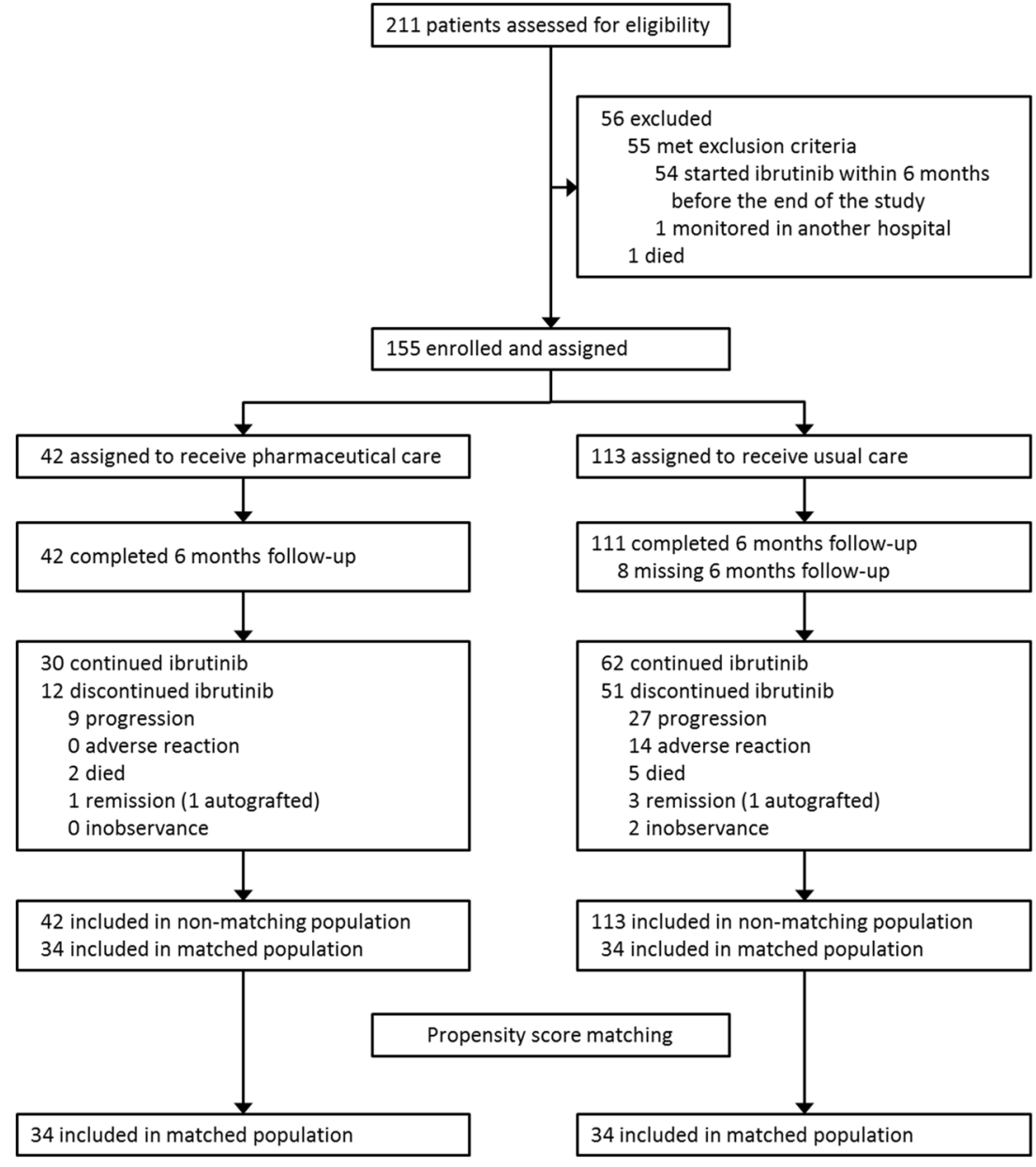

The propensity score was constructed from the prognostic variables of survival, that is, CLL diagnosis, last PFS, time from initial diagnosis, initiation dosage, $\mathrm{LDH}$, comorbidities (other cancer), and drug interactions measured at inclusion. At first, the model was built on complete cases, i.e., in 147 patients, including 42 patients from the PCP group. Difference in both treatment groups can be displayed by the distribution of the PS (Fig. 2), with a c-index at 0.71 . Only 34 (81\%) of the 42 patients from the PCP group could be matched with controls on the basis of their PS, resulting in a matched cohort with reduced imbalances in confounders (Fig. 3), as also illustrated by the c-index at 0.503 .

\section{Efficacy endpoint}

Based on the matched sample, the effect of PCP on PFS was significant $(\mathrm{HR}=0.26 ; 95 \% \mathrm{CI}, 0.11-0.61 ; p=0.002)$ (Table 2). Sensitivity analysis based on average scores from 30 imputed complete datasets of 155 patients did not markedly modify these results; 35 of the 42 treated patients could be matched on their score. Estimated treatment effect was slightly modified (Table 2). The median was not reached at
30 months as compared with a median duration of PFS of 14 months in the usual care group (Fig. 4).

Based on the matched sample, the effect of PCP was significant on time to treatment failure as well on overall survival (Table 2). The PCP significantly prolonged the duration of TTF, with a median not reached at 30 months as compared with a median duration of time to treatment failure of 27 months in the usual care group. At 6 months, $90 \%$ of patients in the PCP group were still treated $(n=38)$, as compared with $60 \%$ in the usual care group $(n=69)$ (Fig. 5). The PCP significantly prolonged the OS, with a median not reached at 30 months as compared with a median OS of 19 months in the usual care group (Fig. 6). Comparison of PFS, TTF, and OS outcomes on the original basis is presented on Supplementary materials.

Filling of the patient diary for self-evaluation of adherence was significantly more respected in PCP group than control group (Table 3). The adherence rate was higher for the PCP group, whether measured by self-evaluation, $99 \%$ vs. $92 \%$ (NS), or by calculation of the MPR, 99\% vs. $90 \%$ (NS). Drug interactions were substantially similar between PCP and control groups at initiation, 1.0 and 0.83 DDI by patient respectively. After 1 month, patients in PCP had fewer DDIs (0.19, i.e., $81 \%$ reduction) while control patients had almost as many drug 
Table 1 Characteristics of the patients at the baseline

\begin{tabular}{|c|c|c|c|c|}
\hline \multirow[b]{2}{*}{ Characteristics $^{\text {a }}$} & \multicolumn{2}{|l|}{ Original cohort } & \multicolumn{2}{|l|}{ Matched cohort } \\
\hline & $\mathrm{PCP}(n=42)$ & Usual care $(n=113)$ & $\mathrm{PCP}(n=34)$ & Usual care $(n=34)$ \\
\hline Age, years - median (IQR) & $66(60.4-73.4)$ & $71(64.5-78.4)$ & $66(59.3-73.4)$ & $69(62.0-77.5)$ \\
\hline Female sex - no. $(\%)$ & $16(38)$ & $51(45)$ & $11(32)$ & $13(38)$ \\
\hline Previous lines of treatment - median (IQR) & $2(1-3)$ & $2(1-3)$ & $2(1-3)$ & $2(1-3)$ \\
\hline Time from last therapy, months - median (IQR) & $15(0-25)$ & $8(0-24)$ & $15(2-25)$ & $3(0-26)$ \\
\hline Time from initial diagnosis, months - median (IQR) & $64(33-109)$ & $76(25-126)$ & $64(38-93)$ & $62(27-105)$ \\
\hline Any comorbidities - no. $(\%)$ & $29(69)$ & $42(37)$ & $\mathrm{N} / \mathrm{A}$ & N/A \\
\hline Cardiovascular comorbidity - no. $(\%)$ & $15(36)$ & $19(17)$ & $12(35)$ & $5(15)$ \\
\hline Other malignancies - no. $(\%)$ & $9(21)$ & $7(6)$ & $3(9)$ & $3(9)$ \\
\hline Comedications - median (IQR) & $4(0-14)$ & $5(0-18)$ & N/A & N/A \\
\hline Chronic lymphocytic leukemia - no. (\%) & $17(40)$ & $63(56)$ & $15(44)$ & $16(47)$ \\
\hline \multicolumn{5}{|l|}{ Binet stage - no. $(\%)^{\mathrm{b}}$} \\
\hline A & 0 & $1(2)$ & 0 & 1 \\
\hline B & $4(24)$ & $4(6)$ & 4 & 1 \\
\hline $\mathrm{C}$ & $13(76)$ & $55(87)$ & 11 & 14 \\
\hline \multicolumn{5}{|l|}{ Rai stage - no. $(\%)^{\mathrm{c}}$} \\
\hline I or II & $9(53)$ & $39(62)$ & 8 & 10 \\
\hline III or IV & $8(47)$ & $24(38)$ & 7 & 6 \\
\hline 17p deletion or TP53 mutation - no. (\%) & $6(14)$ & $29(26)$ & 3 & 6 \\
\hline Mantle cell lymphoma - no. (\%) & $16(38)$ & $16(14)$ & 11 & 8 \\
\hline MIPI - median (IQR) & $6.75(5.9-7.1)$ & $7.6(6.6-8.3)$ & $6.80(5.75-7.40)$ & $7.30(6.15-8.05)$ \\
\hline High & $9(57)$ & $9(57)$ & N/A & N/A \\
\hline Waldenström macroglobulinemia - no. (\%) & $6(14)$ & $17(15)$ & 5 & 3 \\
\hline \multicolumn{5}{|l|}{ ISSWM- no. $(\%)$} \\
\hline Low & $1(17)$ & $3(18)$ & 1 & 1 \\
\hline Intermediate & $1(17)$ & $4(24)$ & 1 & 0 \\
\hline High & $1(17)$ & $4(24)$ & 1 & 1 \\
\hline Diffuse large B cell lymphoma - no. (\%) & $3(7)$ & $10(9)$ & 3 & 6 \\
\hline Hemoglobin, g/dl - median (IQR) & $11.7(10.5-13.3)$ & $10.6(9.4-12.1)$ & $11.9(10.5-13.3)$ & $11.0(10.0-12.2)$ \\
\hline Platelet count, giga/L - median (IQR) & $161(102-225)$ & $125(61-197)$ & $152(101-224)$ & $118(58-164)$ \\
\hline Neutrophil count, giga/L - median (IQR) & $2.9(1.9-4.5)$ & $2.9(1.5-5.1)$ & $3.0(2.0-4.6)$ & $2.8(2.2-4.4)$ \\
\hline $\mathrm{LDH}>\mathrm{ULN}-$ no. $(\%)$ & $17(40)$ & $61(55)$ & $16(47)$ & $16(47)$ \\
\hline Initial dosage of ibrutinib - median (IQR) & $420(420-560)$ & $420(420-420)$ & $420(420-560)$ & $420(420-560)$ \\
\hline
\end{tabular}

$P C P$ pharmaceutical care program, IQR interquartile range, N/A not applicable, MIPI Mantle Cell Lymphoma International Prognostic Index, ISSWM International Prognostic Scoring System for Waldenström macroglobulinemia, $L D H$ lactate dehydrogenase, $U L N$ upper limit normal

${ }^{\text {a }}$ No significant differences between the two groups at baseline. Percentages may not total the overall number in the category because of rounding

${ }^{\mathrm{b}}$ Stage A denotes low-risk disease, stage $\mathrm{B}$ intermediate risk, and stage $\mathrm{C}$ high risk

${ }^{\mathrm{c}}$ Stage I or II denotes intermediate-risk disease, and stage III or IV high risk

interactions as initiation ( 0.77 , i.e., $7.2 \%$ reduction). The proportion of patients with at least one DDI decreased from 64 to $14 \%$ in the PCP group and from 56 to $49 \%$ in the control group.

\section{Safety outcomes}

All 155 patients were evaluated for safety. The most common any grade AEs, defined as those observed in $10 \%$ or more in either group, were infections ( $15 \%$ control, $16 \% \mathrm{PCP}$ ), fatigue (10\% control, 24\% PCP), diarrheas (9\% control, 20\% PCP), hemorrhage events (9\% control, 18\% PCP), and muscle spasms (7\% control, 13\% PCP). Adverse events of any grade were less common in control than PCP patients (NS) (Table 4).

Grade $\geq 3$ AEs occurred in larger proportions for patients in control (15\%) than PCP (8\%) patients. The most common 


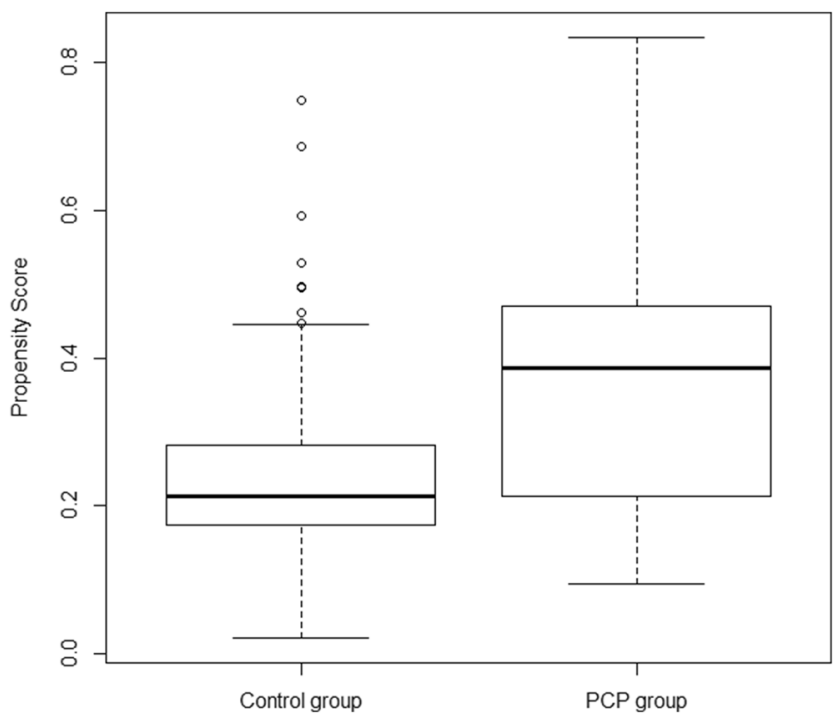

Fig. 2 Comparison of the propensity scores on the original basis (unmatched). Difference in both treatment groups can be visualized by distribution of the propensity score, with a c-index at 0.71 . PCP, pharmaceutical care program grade $\geq 3$ AEs observed was infections ( $5 \%$ control, $1 \% \mathrm{PCP}$ ). Discontinuation of treatment owing to AEs did not occur in the PCP group and occurred for $25 \%$ patients in the control group (in orders of frequency, diarrhea, cardiac disorder, infections, fatigue, and arthralgia).

There have been more therapeutic adaptations of ibrutinib (treatment suspension and/or dose reduction) following side effects in the PCP group (2\% control, $9 \%$ PCP).

\section{Discussion}

This observational study aimed at evaluating the effects of a PCP in patients receiving ibrutinib, assuming that it could increase PFS by reducing dose reduction or treatment discontinuation. To our knowledge, results are the first to date showing a significant improvement of PFS and OS in patients treated by an oral chemotherapy because of a monitoring program, with a significantly delayed treatment failure and a reduction in severe toxicities.

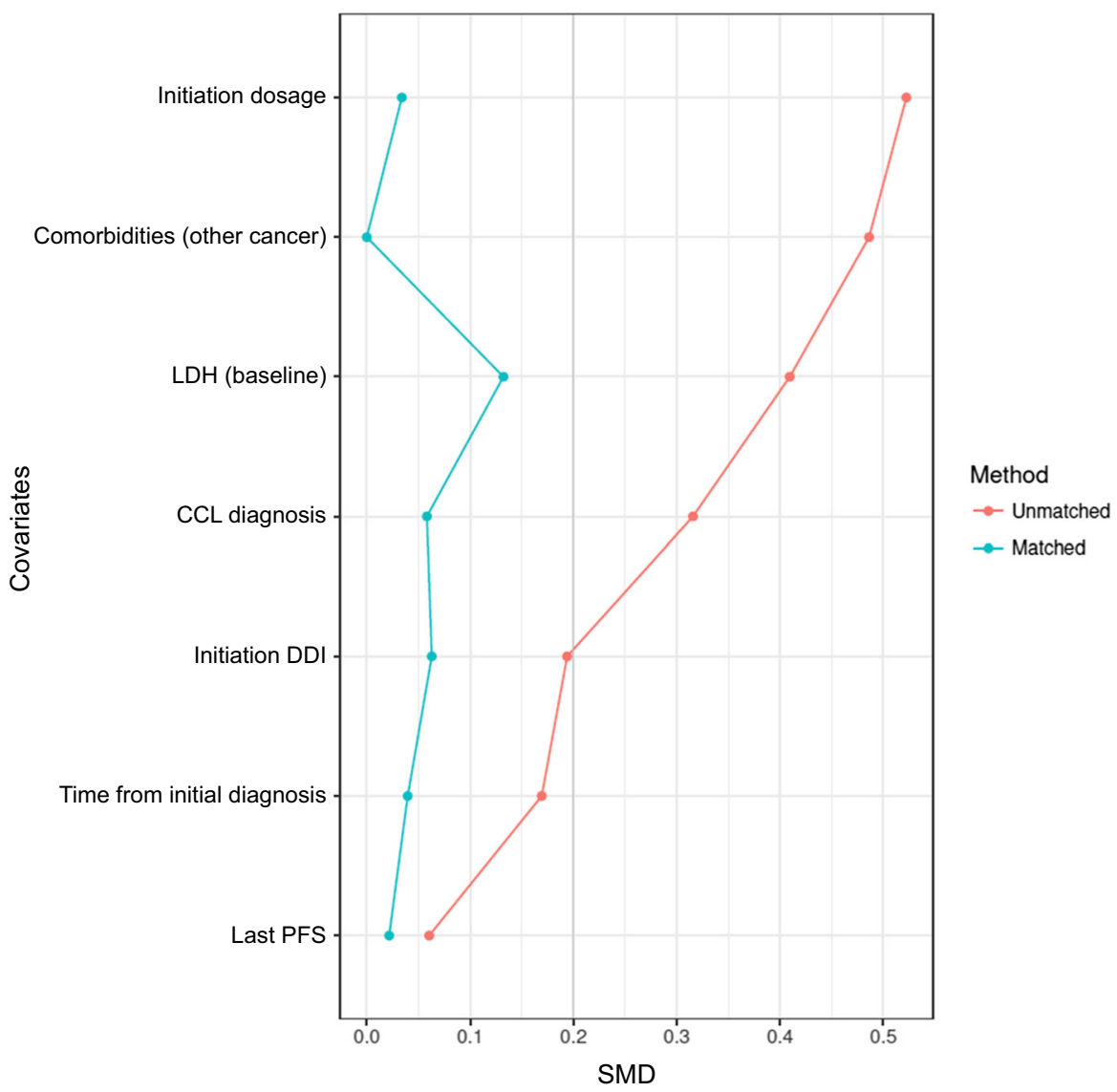

Fig. 3 Comparison of imbalances between groups on the unmatched and matched basis. Matching without replacement on the propensity score within a caliper of 0.1 leads to holding 34 patients from the PCP group on the 42 of the original basis. After this matching, the c-index is reduced at 0.503 , as well as the standardized mean differences. LDH, lactate dehydrogenase. CLL, chronic lymphocytic leukemia. DDI, drug-drug interactions. PFS, progression-free survival. SMD, standardized mean differences 
Table 2 Estimates of the PCP effect on progression-free survival, overall survival, and time to treatment failure before and after matching on propensity scores

\begin{tabular}{|c|c|c|c|c|}
\hline \multirow[t]{2}{*}{ Outcome } & \multicolumn{2}{|l|}{ Before matching } & \multicolumn{2}{|l|}{ After matching } \\
\hline & $\operatorname{HR}(95 \% \mathrm{CI})$ & $p$ value & $\operatorname{HR}(95 \% \mathrm{CI})$ & $p$ value \\
\hline \multicolumn{5}{|c|}{ Primary outcome, progression free survival } \\
\hline Complete cases & $0.43(0.22-0.83)$ & 0.012 & $0.26(0.11-0.61)$ & 0.002 \\
\hline Multiple imputation & N/A & N/A & $0.31(0.13-0.75)$ & 0.009 \\
\hline \multicolumn{5}{|l|}{ Secondary outcomes } \\
\hline Overall survival & $0.44(0.20-1.00)$ & 0.050 & $0.17(0.05-0.58)$ & 0.004 \\
\hline Time to treatment failure & $0.41(0.22-0.77)$ & 0.005 & $0.26(0.12-0.56)$ & 0.0005 \\
\hline
\end{tabular}

$H R$ hazard ratio, $C I$ confidence interval, $N / A$ not applicable
Although the literature provides effective approaches to improving the management of OOA-treated patients, there is no single, formalized, and extrapolatable method yet. In 2013, the American Society of Clinical Oncology (ASCO) and the Oncology Nursing Society (ONS) added a specific component for monitoring of OOA to their safety recommendations [20]. It defined actions that the clinician must perform to educate the patient to adherence and AEs self-management: a treatment plan must be given to the patient, including information about the molecule as well as therapeutic goal. The clinician should provide additional written information regarding the dosing schedules, AEs key symptoms to look for and how to react to these symptoms, treatments to manage non-serious AEs, and possible DDIs. Furthermore, the clinician must assess the level of patient adherence at each consultation. The oncologist often has limited time during medical consultations. The use of other health professionals has proved the ability of multidisciplinary teams included pharmacists and nurses to achieve the expected results [13]. Some multidisciplinary programs involving pharmacists have shown ability to reduce the severity of adverse events and improve adherence [21].

Fig. 4 Comparison of progression-free survival outcomes on the matched basis. The Kaplan-Meier analysis is the probability of progression-free survival in the PCP group as compared with the control group. Median time for progression is not reached at 30 months in the PCP groups as compared with a median time for progression of 14 months in the usual group
Literature data associated non-adherence to ibrutinib with decreased rates of PFS [9]. Other OOAs in hematology have shown that adherence is the main factor of treatment efficacy [22]. We used the combination of two methods of measuring adherence. More expensive methods exist, such as electronic pill dispensers or blood tests, but they did not show superiority to detect poor adhesion [23]. Patients in our program had higher rates of adherence than the control cohort. This improvement is partly due to the educational component of the program. It depends on initial recommendations, ongoing evaluation and reminders from all stakeholders. Inclusion in the program allows the detection of poor compliance prospectively and its rapid correction. Indeed, patients in both cohorts were asked to self-assess their adherence. It was respected for less than half of the patients in the control group, limiting the ability to detect and correct poor adherence.

Our current study further demonstrates a significant improvement in tolerance of patients in PCP cohort. This is mainly the result of three components of the program: patient education to self-management of AEs, transition from hospital to community, and reduction of DDIs. There is evidence that patient education to self-management of AEs has a beneficial impact on

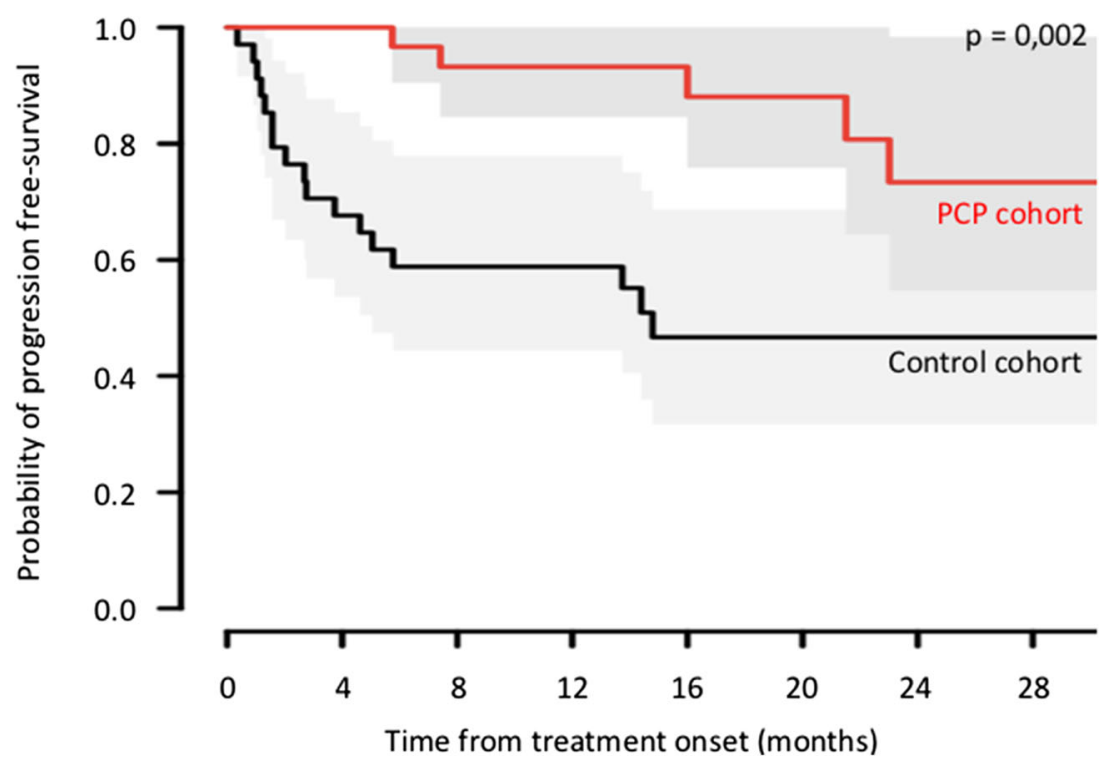


Fig. 5 Comparison of time to treatment failure outcomes on the matched basis. The KaplanMeier analysis is the probability of time to treatment failure in the PCP group as compared with the control group. Median time for time to treatment failure is not reached at 30 months in the PCP groups as compared with a median time for progression of

27 months in the usual group. At 6 months, $90 \%$ of patients in the PCP group were still treated, as compared with $60 \%$ in the usual care group

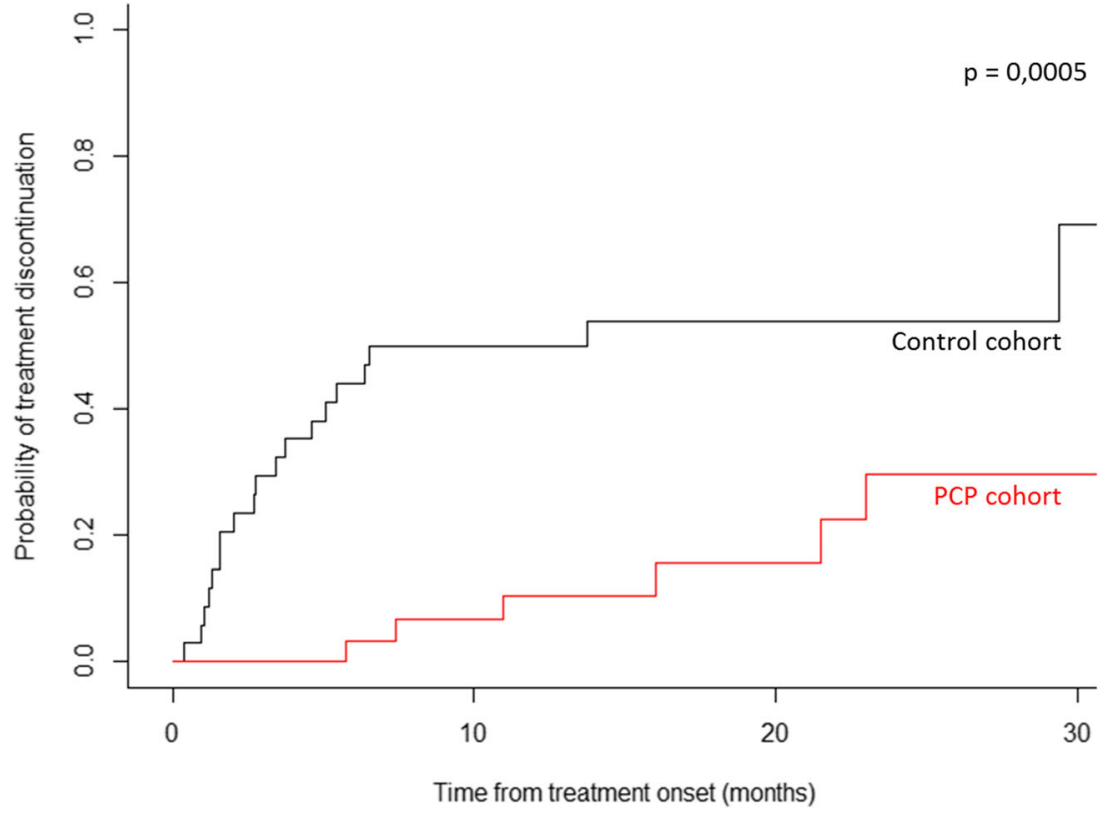

tolerance [24]. In our study, instructions on ibrutinib toxicities were elaborated by a multidisciplinary team involving oncologists, pharmacists, infectiologists, biologists, and nutritionists. These recommendations were evidence-based medicine and concerned digestive, hematological, infectious, cardiovascular, and hemorrhagic events [25-27]. This led to institutional educational materials that were provided to patients in PCP at initiation of treatment. In our study, more low-grade adverse events have been reported in PCP group, which can be explained by improved detection thanks to the intervention. Indeed, the program provides telephone calls by clinical pharmacists and promotion of relations from hospital to community with specialist and general practitioners and community pharmacists. These measures showed that the detection of AEs was improved by
$59 \%$ after 6 months in a pharmaceutical program of education and follow-up of onco-hematologic outpatients [28]. Improving the detectability of toxicities makes it possible to manage it before aggravation. Finally, the prophylaxis of toxicities also depends on the medication-related interventions, in particular when it corrects the DDIs responsible for an increase of ibrutinib in the plasma and prevents the consequent toxicities. At the first prescription of ibrutinib, proportion of patients in both PCP and cohort groups with DDIs were comparable to the literature [16]. The number of DDIs per patient in PCP group was divided by four at 1 month of treatment, lowering to less than 0.2 per patient. All DDIs could not be removed and this led in some cases to a decrease of ibrutinib dosage due to an inevitable association with a strong enzyme inducer.
Fig. 6 Comparison of overall survival outcomes on the matched basis. The Kaplan-Meier analysis is the probability of overall survival in the PCP group as compared with the control group. Median time for survival is not reached at 30 months in the PCP groups as compared with a median time for progression of 19 months in the usual group

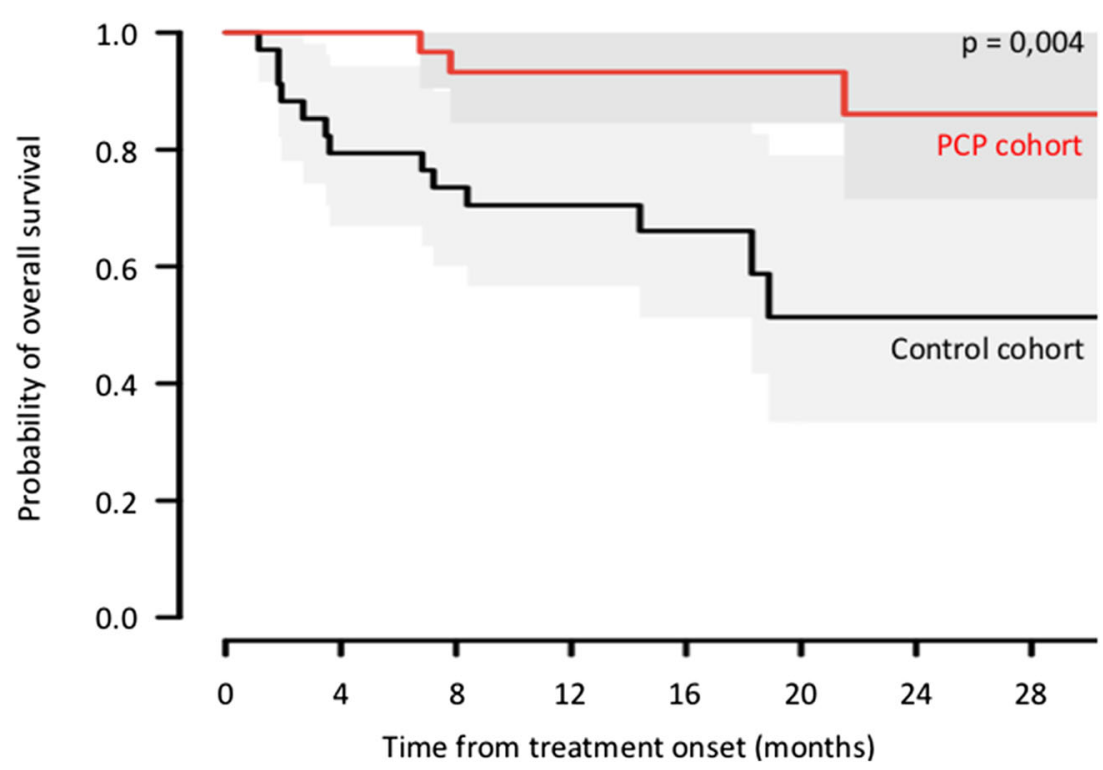


Table 3 Effect of PCP on adherence and drug interactions

\begin{tabular}{lccc}
\hline & PCP & Usual care & $p$ value \\
\hline Adherence & & & \\
Filling of the patient diary (self-evaluation) - no. (\%) & $34(81)$ & $51(45)$ & 0.004 \\
Mean adherence (self-evaluation) - \% (SD) & $99(0.7)$ & $92(23.1)$ & 0.15 \\
Mean adherence (medication possession ratio) - \% (SD) & $99(2.3)$ & $90(25.8)$ & 0.22 \\
Drug interactions & & & \\
DDI by patient at initiation - mean (range) & $1.0(0-4)$ & $0.83(0-5)$ & 0.38 \\
DDI by patient at 1 month - mean (range) & $0.19(0-3)$ & $0.77(0-5)$ & $<0.0001$ \\
\hline
\end{tabular}

$P C P$ pharmaceutical care program, $S D$ standard deviation, $D D I$ drug-drug interaction
Another outcome studied with a direct impact on the effectiveness of ibrutinib was the time to treatment failure. Our results have revealed a significantly increased TTF for patients included in the PCP. A real-world analysis has shown rate of ibrutinib discontinuations due to toxicities range from 12 to $32 \%$ [29]. In our control cohort, the rate of ibrutinib discontinuation attributable to side effects over the entire study period was $25 \%$ versus $0 \%$ in the PCP cohort. As reported in the literature, this can be explained by common dose reductions and temporary treatment interruptions to manage the toxicity early without stopping the treatment permanently [30]. Early detection of adverse effects that allow the multidisciplinary program for patients in PCP cohort lead to more suspensions of treatment, followed or not by a dose adjustment, as compared to the control cohort. Temporary cessations and dose decreases due to adverse events were commonly possible because it does not reduce the long-term efficacy of ibrutinib [10].

Our study has some limitations. First, it was a single-center study based on a sample size of 155 . Second, the two groups were not constituted through randomization but depended on
Table 4 Adverse events, serious adverse events, and ibrutinib dose adjustment

\begin{tabular}{|c|c|c|c|c|}
\hline \multirow[t]{2}{*}{ Adverse event of any grade } & \multicolumn{2}{|c|}{ Event - no. (\%) } & \multicolumn{2}{|c|}{ Ibrutinib dose adjustment - no. ${ }^{a}$} \\
\hline & $\mathrm{PCP}$ & Usual care & PCP & Usual care \\
\hline Fatigue & $42(24)$ & $37(10)$ & 2 & 1 \\
\hline Diarrhea & $34(20)$ & $36(9)$ & 1 & 0 \\
\hline Petechiae and bleeding & $31(18)$ & $36(9)$ & 2 & 1 \\
\hline Infection and infestation & $27(16)$ & $59(15)$ & 4 & 1 \\
\hline Muscle spasm & $23(13)$ & $27(7)$ & 0 & 1 \\
\hline Cardiac disorder & $7(4)$ & $21(5)$ & 1 & 0 \\
\hline Neutropenia & $4(2)$ & $10(3)$ & 6 & 3 \\
\hline Thrombocytopenia & 0 & $11(3)$ & 0 & 0 \\
\hline \multicolumn{5}{|l|}{ Adverse event of grade $\geq 3$} \\
\hline All & $13(8)$ & $57(15)$ & N/A & N/A \\
\hline Neutropenia & $4(2)$ & $8(2)$ & N/A & N/A \\
\hline Fatigue & $3(2)$ & $8(2)$ & N/A & N/A \\
\hline Infection and infestation & $2(1)$ & $20(5)$ & N/A & N/A \\
\hline Diarrhea & $2(1)$ & $8(2)$ & N/A & N/A \\
\hline Cardiac disorder & $1(1)$ & $6(2)$ & N/A & N/A \\
\hline Rash & $1(1)$ & 0 & N/A & N/A \\
\hline Thrombocytopenia & 0 & $3(1)$ & N/A & N/A \\
\hline Petechiae and bleeding & 0 & $3(1)$ & N/A & N/A \\
\hline \multicolumn{5}{|l|}{ Serious adverse event } \\
\hline Neutropenia & $2(1)$ & $2(1)$ & N/A & N/A \\
\hline Cardiac disorder & 0 & $2(1)$ & N/A & N/A \\
\hline Infection and infestation & 0 & $1(0)$ & N/A & N/A \\
\hline Diarrhea & 0 & $1(0)$ & N/A & $\mathrm{N} / \mathrm{A}$ \\
\hline
\end{tabular}

$P C P$ pharmaceutical care program, N/A not applicable

${ }^{\text {a }}$ Dose reduction or suspension of ibrutinib treatment until event correction possibly followed by a dose reduction 
the oncologist decision, so that some selection bias may have occurred; it is likely that the program was mostly proposed to those patients with a past history of intolerance or of poor adherence, or at high risk of adverse events due to comorbidities. Therefore, we used propensity score matching to allow handling such a confounding-by-indication bias. Nevertheless, matching on propensity scores leads to unbiased estimation of treatment effects when certain assumptions hold, notably that all confounding covariates were observed and included in the propensity score; then, this implies the balance generated by propensity score matching leads to unbiased treatment effect estimates. However, we cannot exclude the possibility of unmeasured confounders. Thus, further multicenter randomized studies should confirm the benefits of such a PCP.

\section{Conclusion}

Our program is consistent with the various recommendations concerning OOA. The individualization of the care pathway with adherence and tolerance monitoring, patient education for self-management, and coordination from hospital to community allow a better use of ibrutinib. A pharmaceutical care program provides a personalized environment for outpatient monitoring and control of the key risks associated with OOA. This study shows for the first time evidence that such a program improves adherence and tolerance with ibrutinib leading to longer treatment durations that are directly related to better PFS and OS. Our findings support the need of clinical pharmacists in the oral chemotherapy management.

Authors' contributions Conception and design: Sylvie Chevret, Jeremie Zerbit, Isabelle Madelaine, Catherine Thieblemont.

Provision of study material or patients: Catherine Thieblemont, Isabelle Madelaine.

Collection and assembly of data: Sylvie Chevret, Jeremie Zerbit.

Data analysis and interpretation: Sylvie Chevret, Jeremie Zerbit, Isabelle Madelaine, Catherine Thieblemont.

Manuscript writing: All authors.

Final approval of manuscript: All authors.

Accountable for all aspects of the work: All authors.

Data availability The datasets generated during and/or analyzed during the current study are available from the corresponding author on reasonable request.

\section{Compliance with ethical standards}

Conflict of interest The authors declare that they have no conflict of interest.

Ethics approval Institutional Review Board Agreement of US Department of Health and Human Services No. IRB 00003835. IDRCB: 2017-A03604-49.
Consent to participate Informed consent was obtained from all individual participants included in the study.

Consent for publication Not applicable.

Code availability None.

Open Access This article is licensed under a Creative Commons Attribution 4.0 International License, which permits use, sharing, adaptation, distribution and reproduction in any medium or format, as long as you give appropriate credit to the original author(s) and the source, provide a link to the Creative Commons licence, and indicate if changes were made. The images or other third party material in this article are included in the article's Creative Commons licence, unless indicated otherwise in a credit line to the material. If material is not included in the article's Creative Commons licence and your intended use is not permitted by statutory regulation or exceeds the permitted use, you will need to obtain permission directly from the copyright holder. To view a copy of this licence, visit http://creativecommons.org/licenses/by/4.0/.

\section{References}

1. Burger JA, Tedeschi A, Barr PM, Robak T, Owen C, Ghia P, Bairey O, Hillmen P, Bartlett NL, Li J, Simpson D, Grosicki S, Devereux S, McCarthy H, Coutre S, Quach H, Gaidano G, Maslyak Z, Stevens DA, Janssens A, Offner F, Mayer J, O'Dwyer M, Hellmann A, Schuh A, Siddiqi T, Polliack A, Tam CS, Suri D, Cheng M, Clow F, Styles L, James DF, Kipps TJ, RESONATE-2 Investigators. (2015) Ibrutinib as initial therapy for patients with chronic lymphocytic leukemia. N Engl J Med 373: 2425-2437

2. Treon SP, Tripsas CK, Meid K, Warren D, Varma G, Green R, Argyropoulos KV, Yang G, Cao Y, Xu L, Patterson CJ, Rodig S, Zehnder JL, Aster JC, Harris NL, Kanan S, Ghobrial I, Castillo JJ, Laubach JP, Hunter ZR, Salman Z, Li J, Cheng M, Clow F, Graef T, Palomba ML, Advani RH (2015) Ibrutinib in previously treated Waldenström's macroglobulinemia. N Engl J Med 372:1430-1440

3. Dreyling M, Jurczak W, Jerkeman M, Silva RS, Rusconi C, Trneny M, Offner F, Caballero D, Joao C, Witzens-Harig M, Hess G, Bence-Bruckler I, Cho SG, Bothos J, Goldberg JD, Enny C, Traina S, Balasubramanian S, Bandyopadhyay N, Sun S, Vermeulen J, Rizo A, Rule S (2016) Ibrutinib versus temsirolimus in patients with relapsed or refractory mantle-cell lymphoma: an international, randomised, open-label, phase 3 study. Lancet. 387: 770-778

4. Byrd JC, Brown JR, O'Brien S et al (2014) Ibrutinib versus ofatumumab in previously treated chronic lymphoid leukemia. $\mathrm{N}$ Engl J Med 371:213-223

5. Maddocks KJ, Ruppert AS, Lozanski G, Heerema NA, Zhao W, Abruzzo L, Lozanski A, Davis M, Gordon A, Smith LL, Mantel R, Jones JA, Flynn JM, Jaglowski SM, Andritsos LA, Awan F, Blum KA, Grever MR, Johnson AJ, Byrd JC, Woyach JA (2015) Etiology of ibrutinib therapy discontinuation and outcomes in patients with chronic lymphocytic leukemia. JAMA Oncol. 1:80-87

6. Gustine JN, Meid K, Dubeau T, Severns P, Hunter ZR, Guang Y, $\mathrm{Xu}$ L, Treon SP, Castillo JJ (2018) Ibrutinib discontinuation in Waldenström macroglobulinemia: etiologies, outcomes, and $\operatorname{IgM}$ rebound. Am J Hematol 93:511-517

7. Mato AR, Hill BT, Lamanna N, Barr PM, Ujjani CS, Brander DM, Howlett C, Skarbnik AP, Cheson BD, Zent CS, Pu JJ, Kiselev P, Foon K, Lenhart J, Henick Bachow S, Winter AM, Cruz AL, Claxton DF, Goy A, Daniel C, Isaac K, Kennard KH, Timlin C, Fanning M, Gashonia L, Yacur M, Svoboda J, Schuster SJ, Nabhan 
C (2017) Optimal sequencing of ibrutinib, idelalisib, and venetoclax in chronic lymphocytic leukemia: results from a multicenter study of 683 patients. Ann Oncol 28:1050-1056

8. Bassan F, Peter F, Houbre B, Brennstuhl MJ, Costantini M, Speyer E, Tarquinio C (2014) Adherence to oral antineoplastic agents by cancer patients: definition and literature review. Eur J Cancer Care 23:22-35

9. Barr PM, Brown JR, Hillmen P, O'Brien S, Barrientos JC, Reddy NM, Coutre S, Mulligan SP, Jaeger U, Furman RR, Cymbalista F, Montillo M, Dearden C, Robak T, Moreno C, Pagel JM, Burger JA, Suzuki S, Sukbuntherng J, Cole G, James DF, Byrd JC (2017) Impact of ibrutinib dose adherence on therapeutic efficacy in patients with previously treated CLL/SLL. Blood. 129:2612-2615

10. Ahn IE, Basumallik N, Tian X, Soto SJ, Wiestner A (2019) Clinicallyindicated ibrutinib dose interruptions and reductions do not compromise long-term outcomes in CLL. Blood. 133:2452-2455

11. de Zwart L, Snoeys J, De Jong J, Sukbuntherng J, Mannaert E, Monshouwer M (2016) Ibrutinib dosing strategies based on interaction potential of CYP3A4 perpetrators using physiologically based pharmacokinetic modeling. Clin Pharmacol Ther 100:548-557

12. Finnes HD, Chaffee KG, Call TG, Ding W, Kenderian SS, Bowen DA, Conte M, McCullough KB, Merten JA, Bartoo GT, Smith MD, Leis J, Chanan-Khan A, Schwager SM, Slager SL, Kay NE, Shanafelt TD, Parikh SA (2017) Pharmacovigilance during ibrutinib therapy for chronic lymphocytic leukemia (CLL)/small lymphocytic lymphoma (SLL) in routine clinical practice. Leuk Lymphoma 58:1376-1383

13. Zerillo JA, Goldenberg BA, Kotecha RR, Tewari AK, Jacobson JO, Krzyzanowska MK (2018) Interventions to improve oral chemotherapy safety and quality: a systematic review. JAMA Oncol 4: $105-117$

14. Van Leeuwen RWF, Brundel DHS, Neef C et al (2013) Prevalence of potential drug-drug interactions in cancer patients treated with oral anticancer drugs. Br J Cancer 108:1071-1078

15. Austin PC (2014) The use of propensity score methods with survival or time-to-event outcomes: reporting measures of effect similar to those used in randomized experiments. Stat Med 33:1242-1258

16. Little RJ, Rubin DB (2000) Causal effects in clinical and epidemiological studies via potential outcomes: concepts and analytical approaches. Annu Rev Public Health 21:121-145

17. Franklin JM, Rassen JA, Ackermann D, Bartels DB, Schneeweiss S (2014) Metrics for covariate balance in cohort studies of causal effects. Stat Med 33:1685-1699

18. Gayat E, Resche-Rigon M, Mary J-Y, Porcher R (2012) Propensity score applied to survival data analysis through proportional hazards models: a Monte Carlo study. Pharm Stat 11:222-229

19. Leyrat C, Seaman SR, White IR, Douglas I, Smeeth L, Kim J, Resche-Rigon M, Carpenter JR, Williamson EJ (2019) Propensity score analysis with partially observed covariates: how should multiple imputation be used? Stat Methods Med Res 28:3-19

20. Neuss MN, Polovich M, McNiff K et al (2013) 2013 Updated American Society of Clinical Oncology/Oncology Nursing Society chemotherapy administration safety standards including standards for the safe administration and Management of Oral Chemotherapy. J Oncol Pract 9:5s-13s
21. Segal EM, Bates J, Fleszar SL et al (2019) Demonstrating the value of the oncology pharmacist within the healthcare team. J Oncol Pharm Pract 25:1945-1967

22. Noens L, van Lierde M-A, De Bock R et al (2009) Prevalence, determinants, and outcomes of nonadherence to imatinib therapy in patients with chronic myeloid leukemia: the ADAGIO study. Blood. 113:5401-5411

23. Mislang AR, Wildes TM, Kanesvaran R, Baldini C, Holmes HM, Nightingale G, Coolbrandt A, Biganzoli L (2017) Adherence to oral cancer therapy in older adults: the International Society of Geriatric Oncology (SIOG) taskforce recommendations. Cancer Treat Rev 57:58-66

24. Molassiotis A, Brearley S, Saunders M, Craven O, Wardley A, Farrell C, Swindell R, Todd C, Luker K (2009) Effectiveness of a home care nursing program in the symptom management of patients with colorectal and breast cancer receiving oral chemotherapy: a randomized, controlled trial. J Clin Oncol 27:6191-6198

25. de Weerdt I, Koopmans SM, Kater AP, van Gelder M (2017) Incidence and management of toxicity associated with ibrutinib and idelalisib: a practical approach. Haematologica. 102:1629 1639

26. Lipsky AH, Farooqui MZH, Tian X, Martyr S, Cullinane AM, Nghiem K, Sun C, Valdez J, Niemann CU, Herman SEM, Saba N, Soto S, Marti G, Uzel G, Holland SM, Lozier JN, Wiestner A (2015) Incidence and risk factors of bleeding-related adverse events in patients with chronic lymphocytic leukemia treated with ibrutinib. Haematologica. 100:1571-1578

27. Chai KL, Rowan G, Seymour JF, Burbury K, Carney D, Tam CS (2017) Practical recommendations for the choice of anticoagulants in the management of patients with atrial fibrillation on ibrutinib. Leuk Lymphoma. 58:2811-2814

28. Ribed A, Romero-Jiménez RM, Escudero-Vilaplana V, IglesiasPeinado I, Herranz-Alonso A, Codina C, Sanjurjo-Sáez M (2016) Pharmaceutical care program for onco-hematologic outpatients: safety, efficiency and patient satisfaction. Int J Clin Pharm 38:280-288

29. Mato AR, Nabhan C, Thompson MC, Lamanna N, Brander DM, Hill B, Howlett C, Skarbnik A, Cheson BD, Zent C, Pu J, Kiselev P, Goy A, Claxton D, Isaac K, Kennard KH, Timlin C, Landsburg D, Winter A, Nasta SD, Bachow SH, Schuster SJ, Dorsey C, Svoboda J, Barr P, Ujjani CS (2018) Toxicities and outcomes of 616 ibrutinib-treated patients in the United States: a real-world analysis. Haematologica. 103:874-879

30. Winqvist M, Andersson P-O, Asklid A, Karlsson K, Karlsson C, Lauri B, Lundin J, Mattsson M, Norin S, Sandstedt A, Rosenquist R, Späth F, Hansson L, Österborg A, for the Swedish CLL Group (2019) Long-term real-world results of ibrutinib therapy in patients with relapsed or refractory chronic lymphocytic leukemia: 30month follow up of the Swedish compassionate use cohort. Haematologica. 104:e208-e210

Publisher's note Springer Nature remains neutral with regard to jurisdictional claims in published maps and institutional affiliations. 